\title{
Correction to: The genome sequence of the grape phylloxera provides insights into the evolution, adaptation, and invasion routes of an iconic pest
}

Claude Rispe ${ }^{1 *}{ }^{*}$, Fabrice Legeai ${ }^{2^{*}}$, Paul D. Nabity ${ }^{3}$, Rosa Fernández ${ }^{4,5}$, Arinder K. Arora ${ }^{6}$, Patrice Baa-Puyoulet ${ }^{7}$, Celeste R. Banfill ${ }^{8}$, Leticia Bao ${ }^{9}$, Miquel Barberà ${ }^{10}$, Maryem Bouallègue ${ }^{11}$, Anthony Bretaudeau ${ }^{2}$, Jennifer A. Brisson ${ }^{12}$, Federica Calevro ${ }^{7}$, Pierre Capy ${ }^{13}$, Olivier Catrice ${ }^{14}$, Thomas Chertemps ${ }^{15}$, Carole Couture $^{16}$, Laurent Delière ${ }^{16}$, Angela E. Douglas ${ }^{6,17}$, Keith Dufault-Thompson ${ }^{18}$, Paula Escuer ${ }^{19}$, Honglin Feng ${ }^{20,21}$, Astrid Forneck ${ }^{22}$, Toni Gabaldón ${ }^{4,23,24}$, Roderic Guigó ${ }^{25,26}$, Frédérique Hilliou ${ }^{27}$, Silvia Hinojosa-Alvarez ${ }^{19}$, Yi-min Hsiao ${ }^{28,29}$, Sylvie Hudaverdian ${ }^{30}$, Emmanuelle Jacquin-Joly ${ }^{31}$, Edward B. James ${ }^{8}$, Spencer Johnston ${ }^{32}$, Benjamin Joubard ${ }^{16}$, Gaëlle Le Goff ${ }^{33}$, Gaël Le Trionnaire ${ }^{30}$, Pablo Librado ${ }^{34}$, Shanlin Liư ${ }^{35,36,37}$, Eric Lombaert ${ }^{38}$, Hsiao-ling Lu ${ }^{39}$, Martine Maïbèche ${ }^{15}$, Mohamed Makni ${ }^{11}$, Marina Marcet-Houben ${ }^{4}$, David Martínez-Torres ${ }^{40}$, Camille Meslin ${ }^{31}$, Nicolas Montagné ${ }^{41}$, Nancy A. Moran ${ }^{42}$, Daciana Papura ${ }^{16}$, Nicolas Parisot ${ }^{7}, Y^{2}$ van Rahbé ${ }^{43}$, Mélanie Ribeiro Lopes ${ }^{7}$, Aida Ripoll-Cladellas ${ }^{25}$, Stéphanie Robin ${ }^{44}$, Céline Roques ${ }^{45}$, Pascale Roux ${ }^{16}$, Julio Rozas ${ }^{19}$, Alejandro Sánchez-Gracia ${ }^{19}$, Jose F. Sánchez-Herrero ${ }^{19}$, Didac Santesmasses ${ }^{25,46}$, Iris Scatoni ${ }^{47}$, Rémy-Félix Serre ${ }^{45}$, Ming Tang ${ }^{37}$, Wenhua Tian ${ }^{3}$, Paul A. Umina ${ }^{48}$, Manuella van Munster ${ }^{49}$, Carole Vincent-Monégat ${ }^{7}$, Joshua Wemmer ${ }^{3}$, Alex C. C. Wilson ${ }^{8}$, Ying Zhang ${ }^{18}$, Chaoyang Zhao ${ }^{3}$, Jing Zhao ${ }^{35,36}$, Serena Zhao ${ }^{42}$, Xin Zhou ${ }^{37}$, François Delmotte ${ }^{16^{*}+}$ and Denis Tagu ${ }^{30^{*}+}$

\section{Correction to: BMC Biol 18, 90 (2020)} https://doi.org/10.1186/s12915-020-00820-5

Following publication of the original article [1], the authors identified an error in Fig. 4. The error was made in the $\mathrm{x}$-axis labels of the three panels, which were noted as "pairwise $\mathrm{dN} / \mathrm{dS}$ ". The figure represents distributions of $\mathrm{dS}$, so the labels were corrected as "pairwise $\mathrm{dS}$ ". The correct Fig. 4 is given below.

The original article can be found online at https://doi.org/10.1186/s12915020-00820-5.

\footnotetext{
* Correspondence: claude.rispe@inrae.fr; fabrice.legeai@inrae.fr; francois.delmotte@inrae.fr; denis.tagu@inrae.fr

${ }^{+}$Claude Rispe and Fabrice Legeai are co-first authors.

${ }^{\dagger}$ François Delmotte and Denis Tagu are co-last authors.

${ }^{1}$ BIOEPAR, INRAE, Oniris, Nantes, France

²BIPAA, IGEPP, Agrocampus Ouest, INRAE, Université de Rennes 1, 35650 Le

Rheu, France

${ }^{16}$ SAVE, INRAE, Bordeaux Sciences Agro, Villenave d'Ornon, France

${ }^{30}$ IGEPP, Agrocampus Ouest, INRAE, Université de Rennes 1, 35650 Le Rheu, France

Full list of author information is available at the end of the article
}

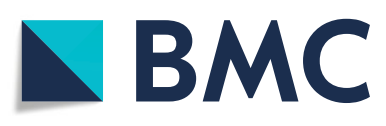

(c) The Author(s). 2020 Open Access This article is licensed under a Creative Commons Attribution 4.0 International License, which permits use, sharing, adaptation, distribution and reproduction in any medium or format, as long as you give appropriate credit to the original author(s) and the source, provide a link to the Creative Commons licence, and indicate if changes were made. The images or other third party material in this article are included in the article's Creative Commons licence, unless indicated otherwise in a credit line to the material. If material is not included in the article's Creative Commons licence and your intended use is not permitted by statutory regulation or exceeds the permitted use, you will need to obtain permission directly from the copyright holder. To view a copy of this licence, visit http://creativecommons.org/licenses/by/4.0/ The Creative Commons Public Domain Dedication waiver (http://creativecommons.org/publicdomain/zero/1.0/) applies to the data made available in this article, unless otherwise stated in a credit line to the data. 

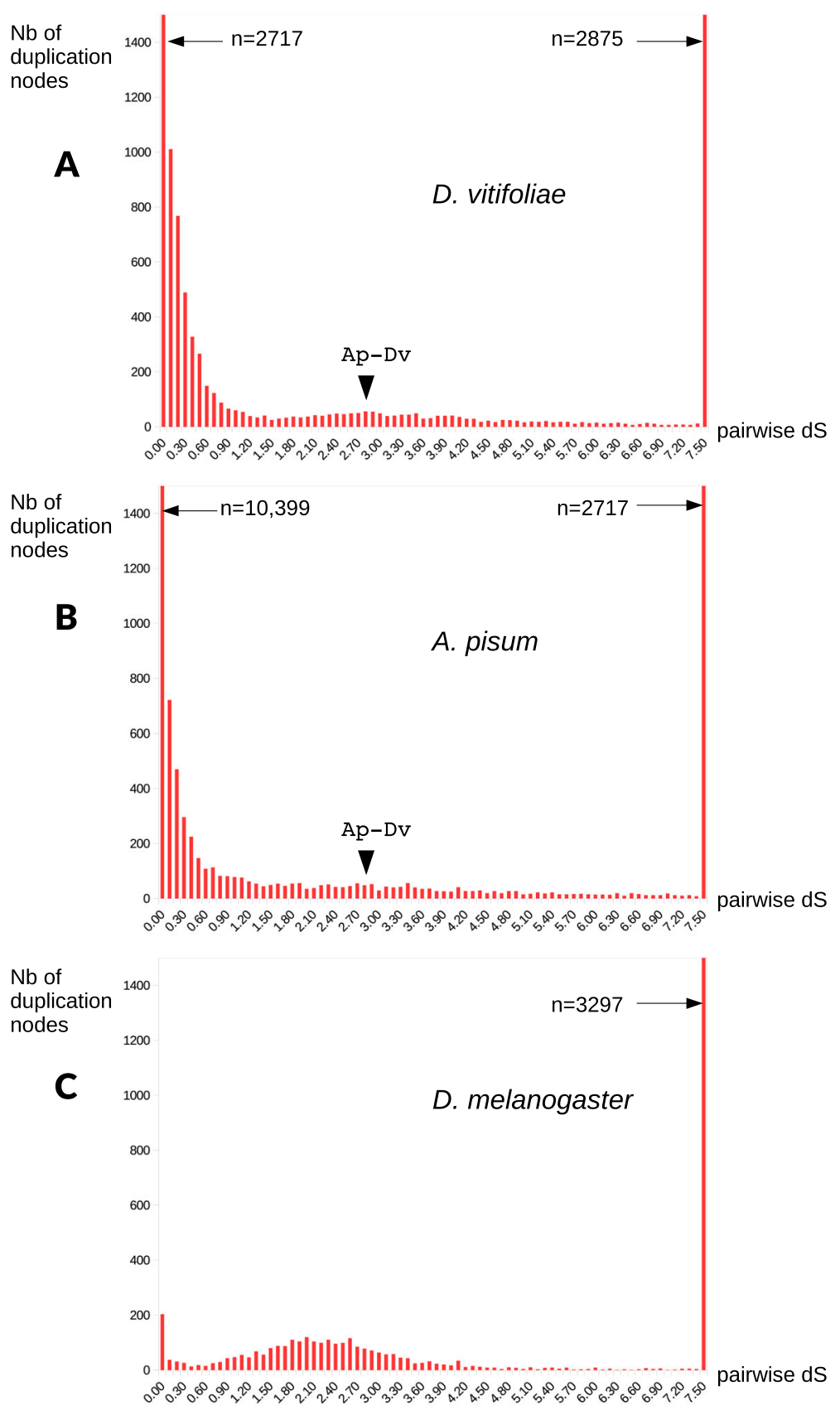

Fig. 4 Distribution of synonymous distances among paralogs for grape vine phylloxera (panel a, D. vitifoliae), pea aphid (panel b, A. pisum), and fruit fly (panel c, D. melanogaster). Paralogs were identified as RBH pairs, with an iterative approach allowing to cover both recent duplications (terminal nodes in gene families) and more ancient duplications (internal nodes). For readability, the $y$-axis (number of dS classes) is truncated to 1500 (numbers above that threshold are indicated on the figures). For both A. pisum and D. vitifoliae, an arrowhead indicates the median dS between orthologs (RBH genes between the two species), $\mathrm{dS}=2.83$ : this metric, a proxy of the age of separation between the two species allows to distinguish duplications that are more recent (left of the arrow, lower dS values) *or more ancient (right of the arrowhead, higher dS) than the speciation event 


\section{Author details}

'BIOEPAR, INRAE, Oniris, Nantes, France. ${ }^{2}$ BIPAA, IGEPP, Agrocampus Ouest, INRAE, Université de Rennes 1, 35650 Le Rheu, France. ${ }^{3}$ Department of Botany and Plant Sciences, University of California, Riverside, USA. ${ }^{4}$ Bioinformatics and Genomics Unit, Centre for Genomic Regulation (CRG), Barcelona Institute of Science and Technology, Dr. Aiguader, 88, 08003 Barcelona, Spain. ${ }^{5}$ Present address: Institute of Evolutionary Biology (CSIC-UPF), Passeig marítim de la Barceloneta 37-49, 08003 Barcelona, Spain ${ }^{6}$ Department of Entomology, Cornell University, Ithaca, NY 14853, USA. ${ }^{7}$ Univ Lyon, INSA-Lyon, INRAE, BF2I, UMR0203, F-69621 Villeurbanne, France. ${ }^{8}$ Department of Biology, University of Miami, Coral Gables, FL 33146, USA. ${ }^{9}$ Facultad de Agronomía, Montevideo, Uruguay. ${ }^{10}$ Institut de Biologia Integrativa de Sistemes, Parc Cientific Universitat de Valencia, C/ Catedrático José Beltrán n² 2, 46980 Paterna, València, Spain. ${ }^{11}$ Université de Tunis El Manar, Faculté des Sciences de Tunis, LR01ES05 Biochimie et Biotechnologie, 2092 Tunis, Tunisia. ${ }^{12}$ Department Biol, Univ Rochester, Rochester, NY 14627, USA. ${ }^{13}$ Laboratoire Evolution, Génomes, Comportement, Ecologie CNRS, Univ. Paris-Sud, IRD, Université Paris-Saclay, Gif-sur-Yvette, France. ${ }^{14}$ LIPM, Université de Toulouse, INRAE, CNRS, Castanet-Tolosan, France. ${ }^{15}$ Sorbonne Université, UPEC, Université Paris 7, INRAE, CNRS, IRD, Institute of Ecology and Environmental Sciences, Paris, France. ${ }^{16}$ SAVE, INRAE, Bordeaux Sciences Agro, Villenave d'Ornon, France. ${ }^{17}$ Department of Molecular Biology and Genetics, Cornell University, Ithaca, NY 14853, USA. ${ }^{18}$ Department of Cell and Molecular Biology, College of the Environment and Life Sciences, University of Rhode Island, Kingston, RI, USA. ${ }^{19}$ Departament de Genètica, Microbiologia i Estadística and Institut de Recerca de la Biodiversitat (IRBio), Universitat de Barcelona, 08028 Barcelona, Spain. ${ }^{20}$ Department of Biology, University of Miami, Coral Gables, USA. ${ }^{21}$ Current affiliation: Boyce Thompson Institute for Plant Research, Cornell University, Ithaca, USA. ${ }^{22}$ Universität für Bodenkultur (BOKU), Vienna, Austria. ${ }^{23}$ Universitat Pompeu Fabra, 08003 Barcelona, Spain. ${ }^{24}$ Institució Catalana de Recerca i Estudis Avançats (ICREA), Pg. Lluís Companys 23, 08010 Barcelona, Spain. ${ }^{25}$ Centre for Genomic Regulation (CRG), The Barcelona Institute of Science and Technology, Barcelona, Spain. ${ }^{26}$ Universitat Pompeu Fabra (UPF), Barcelona, Spain. ${ }^{27}$ Université Côte d'Azur, INRAE, CNRS, Institut Sophia Agrobiotech, Sophia-Antipolis, France. ${ }^{28}$ Institute of Biotechnology and Department of Entomology, College of Bioresources and Agriculture, National Taiwan University, Taipei, Taiwan. ${ }^{29}$ Present affiliation: Bone and Joint Research Center, Chang Gung Memorial Hospital, Taoyuan, Taiwan. ${ }^{30}$ IGEPP, Agrocampus Ouest, INRAE, Université de Rennes 1 , 35650 Le Rheu, France. ${ }^{31}$ INRAE, Institute of Ecology and Environmental Sciences, Versailles, France. ${ }^{32}$ Department of Entomology, Texas A\&M University, College Station, TX 77843, USA. ${ }^{33}$ Université Côte d'Azur, INRAE, CNRS, Institut Sophia Agrobiotech, Sophia-Antipolis, France. ${ }^{34}$ Laboratoire d'Anthropobiologie Moléculaire et d'Imagerie de Synthèse, CNRS UMR 5288, Université de Toulouse, Université Paul Sabatier, Toulouse, France. ${ }^{35} \mathrm{China}$ National GeneBank-Shenzhen, BGI-Shenzhen, Shenzhen 518083, Guangdong Province, People's Republic of China. ${ }^{36} \mathrm{BGI}-S h e n z h e n$, Shenzhen 518083, Guangdong Province, People's Republic of China. ${ }^{37}$ Department of Entomology, College of Plant Protection, China Agricultural University, Beijing 100193, People's Republic of China. ${ }^{38}$ Université Côte d'Azur, INRAE, CNRS, ISA, Sophia Antipolis, France. ${ }^{39}$ Department of Post-Modern Agriculture, MingDao University, Changhua, Taiwan. ${ }^{40}$ Institut de Biologia Integrativa de Sistemes, Parc Cientific Universitat de Valencia, C/ Catedrático José Beltrán nº 2, 46980 Paterna, València, Spain. ${ }^{41}$ Sorbonne Université, Institute of Ecology and Environmental Sciences, Paris, France. ${ }^{42}$ Department of Integrative Biology, University of Texas at Austin, Austin, USA. ${ }^{43}$ Univ Lyon, INRAE, INSA-Lyon, CNRS, UCBL, UMR5240 MAP, F-69622 Villeurbanne, France. ${ }^{44}$ BIPAA IGEPP, Agrocampus Ouest, INRAE, Université de Rennes 1, 35650 Le Rheu, France. ${ }^{45}$ Plateforme Génomique GeT-PlaGe, Centre INRAE de Toulouse Midi-Pyrénées, 24 Chemin de Borde Rouge, Auzeville, CS 52627, 31326 Castanet-Tolosan Cedex, France. ${ }^{46}$ Division of Genetics, Department of Medicine, Brigham and Women's Hospital, Harvard Medical School, Boston,
MA 02115, USA. ${ }^{47}$ Facultad de Agronoía, Montevideo, Uruguay. ${ }^{48}$ School of BioSciences, The University of Melbourne, Parkville, VIC, Australia. ${ }^{49} \mathrm{BGPI}$, Université Montpellier, CIRAD, INRAE, Montpellier SupAgro, Montpellier, France.

Published online: 11 September 2020

\section{Reference}

1. Rispe $C_{\text {, et }}$ al. The genome sequence of the grape phylloxera provides insights into the evolution, adaptation, and invasion routes of an iconic pest. BMC Biol. 2020;18:90. https://doi.org/10.1186/s12915-020-00820-5. 